\title{
The impact of direct-to-consumer marketing of cancer genetic testing on women according to their genetic risk
}

Jan T. Lowery, PhD, MPH $H^{1}$, Tim Byers, MD, MPH ${ }^{1}$, Lisen Axell, MS, CGC $C^{2}$, Lisa Ku, MS, $C G C^{2}$, and Jillian Jacobellis, $P h D^{3}$

\begin{abstract}
Purpose: To assess the impact of direct-to-consumer marketing for genetic testing among women of varying genetic risk for breast and ovarian cancer. Methods: Telephone surveys were conducted with 315 women in Denver, Colorado, one target audience for the Myriad BRACAnalysis ad campaign. Genetic risk was determined from personal and family history and grouped by probability of having a BRCA1/2 mutation (low $<5 \%$, moderate $5-<10 \%$, high $\geq 10 \%$ ). Results: High-risk women were more knowledgeable about BRACAnalysis and more likely to recall the media ads than were low-risk women ( 60 vs. $39 \%, P<0.01$ ). After seeing the ads, about $40 \%$ of women were more interested in testing and about $10 \%$ expressed increased worry about developing breast or ovarian cancer. Women across all risk groups overstated the benefits and appropriateness of testing. An equal percentage of high- and low-risk women (51 and $60 \%$ ) felt that they would benefit from genetic testing. Conclusion: The campaign effectively reached a large audience. Concern about breast cancer was not appreciably increased. A large percentage of low-risk women (not candidates for testing) expressed interest in testing, suggesting the campaign was too broad. A campaign targeted at high-risk women, who may benefit from testing might be preferred.
\end{abstract} Genet Med 2008:10(12):888-894.

Key Words: direct-to-consumer marketing, genetic testing, BRACAnalysis, BRCA1, BRCA2

Scientific and technologic advances have enabled us to identify individuals who are genetically predisposed to cancer. Although it is appropriate to inform individuals who seek information about their genetic risk in clinical settings, it is not yet clear how to best educate the general population about cancer genetic testing. Direct-to-consumer advertising for pharmaceuticals is now commonplace in this country, but the potential ratio of benefit to risk of direct-to-consumer marketing of cancer genetic testing is unknown.

In September 2002, Myriad Genetics launched a pilot consumer awareness campaign in the Denver, Colorado, and Atlanta, Georgia media markets to promote awareness of BRACAnalysis, a genetic test for inherited mutations in the BRCA1 and $B R C A 2$ genes that substantially increase risk for breast and ovarian cancer. ${ }^{1}$ Mutations in BRCA1 and BRCA2 account for about $4 \%$ of all breast cancers and $8 \%$ of all ovarian cancers. ${ }^{2-4}$ The lifetime risk for breast and ovarian cancer among mutation carriers is about $80 \%$ and $40 \%$, respectively when com-

\footnotetext{
From the ${ }^{1}$ Department of Epidemiology, Colorado School of Public Health-Denver, Aurora, Colorado; ${ }^{2}$ University of Colorado Cancer Center, Aurora, Colorado; and the ${ }^{3}$ Colorado Department of Public Health and Environment, Denver, Colorado.

Jan T. Lowery, PhD, MPH, University of Colorado Denver, School of Public Health, Mail Stop F-538, P.O. Box 6508, Aurora, CO 80045-0508. E-mail: Jan.Lowery@ucdenver.edu.

Disclosure: The authors declare no conflicts of interest.

Submitted for publication June 4, 2008.

Accepted for publication September 11, 2008.

DOI: $10.1097 /$ GIM.0b013e31818de6d7
}

pared with $12 \%$ and $1 \%$ in noncarriers. ${ }^{5,6}$ The campaign consisted of television, radio, and print ads over a 5-month period that were intended to increase awareness about BRACAnalysis and to encourage women to speak with their health care providers about genetic testing for breast and ovarian cancer. Myriad defined the target population for the campaign as women aged 25-54 who had a relative with breast or ovarian cancer.

To assess the impact of the Myriad campaign on the general population, the Centers for Disease Control (CDC) conducted a postmarket survey of women living within the two target areas (Denver and Atlanta) and two control areas (RaleighDurham, NC and Seattle, WA). ${ }^{7}$ The CDC survey was conducted by telephone on a random sample of the female population in the marketing areas and captured information regarding personal risk perception, knowledge, and beliefs about genetic testing and BRACAnalysis, and the potential impacts of the ads. The survey was administered on a random sample of the population designed to assess attitudes about BRCA testing principally among women who would not be regarded as eligible for this genetic test as the prevalence of these mutations in the general population is rare. It is estimated that only 5 of every 1000 women has an inherited mutation in BRCA1 or BRCA2. ${ }^{8}$

To assess whether the campaign may have differentially impacted women at increased risk for genetic predisposition, we conducted a parallel study to accompany the CDC survey in the Denver market area to measure awareness and impact of the me- 
dia campaign in a population of women with known familial risk for breast and ovarian cancer. We sought to compare the impact of the Myriad ad campaign on the knowledge and beliefs about genetic testing among women at average risk and among women at increased risk for having a BRCA mutation.

\section{MATERIALS AND METHODS}

\section{Study population}

Our study population consisted of women enrolled in the Cancer Genetics Network (CGN) at the University of Colorado Cancer Center. The CGN is an National Cancer Institutefunded national registry of families affected by various types of cancer ${ }^{9,10}$; the University of Colorado is 1 of 14 participating CGN sites. The Colorado CGN represents a population-based sample of individuals with varying familial risk for cancer. For the CGN, individuals diagnosed with cancers of the breast, ovary, colon, lung, or prostate in Colorado between 1999 and 2000 were identified by hospital tumor registries and the Colorado Central Cancer Registry. These individuals were contacted by mail and asked to complete a brief family history survey. Based on this information, individuals were invited to participate in the CGN. Individuals with family history of cancer were oversampled for inclusion in the registry but approximately $30 \%$ of cases without positive histories were also sampled and enrolled. In addition to enrolling affected individuals, unaffected, first-degree relatives of cases that enrolled in CGN were also invited to participate.

For the current study, women aged 25-54 who were enrolled in the Colorado CGN, had been diagnosed with breast or ovarian cancer in 1999 or 2000 or were unaffected but had a firstdegree relative who had been diagnosed with breast, ovarian, colon, or prostate cancer during this time, were included. Eligible women must also have been living within the 5-county Denver Metro Area during the Myriad media campaign (September 2002-February 2003). Based on these criteria, a total of 492 eligible women were identified and invited to partake in the survey.

\section{Survey instrument}

We used the survey instrument developed by the CDC for their random population survey, modified slightly to accommodate our study population. ${ }^{7}$ Because personal and family history of cancer for our population was known, we omitted these questions from our survey. We also omitted questions about perceived risk and worry about developing cancer for women known to have had breast or ovarian cancer. With the exception of these omissions, the wording of the remaining questions was not changed to preserve comparability of data across study populations.

Eligible subjects were contacted first by mail to introduce the study and then by telephone to administer the survey. The surveys were conducted over a period of 3 months, approximately $8-10$ months after the Myriad campaign was launched. Telephone surveys took an average of 15 minutes to complete. Interviewers were trained to assure standardization of the sur- vey responses. A protocol was also implemented for establishing contact and for tracking subjects with invalid contact information. Multiple attempts were made to contact subject at various times of the day. Survey data were linked with family history and personal medical history information previously provided by participants as part of their enrollment into the CGN.

\section{Risk of mutation}

Women were categorized into one of three risk groups defined by the probability of having a BRCA mutation. Probabilities were estimated using Mutation Prevalence Tables produced by Myriad Genetic Laboratories ${ }^{11}$ in conjunction with family history information previously provided by participants upon enrollment into the CGN including affected status of the individual, number of breast and/or ovarian cancers in the family (up to second-degree relatives), the ages of diagnoses, and Jewish ancestry. Women with $10 \%$ or greater probability of carrying a mutation were considered to be "high risk," probabilities between $5 \%$ and $10 \%$ were considered "moderate risk," and probabilities of $<5 \%$ were considered "low risk." Mutation risk of $10 \%$ or greater was selected as a cut point for high risk, as this corresponds to the probability of carrying a mutation for any of criteria described as high risk and recommended for genetic testing in the 2008 National Comprehensive Cancer Network (NCCN) guidelines. ${ }^{12}$

Comparison of responses across groups was performed using $\chi^{2}$ test of proportions. All analyses were done using SAS statistical analysis software (SAS Institute, Cary, NC). This study was approved by the Colorado Multiple Institutional Review Board (COMIRB), and informed consent was obtained from all participants.

\section{RESULTS}

Of the 492 women invited to participate, 315 women completed the telephone survey (64\% response). We were unable to contact 96 women within the survey period (of 3 months) and 80 women refused participation. The median age of respondents was 45 years; the majority was non-Hispanic white (94\%) and had more than a high-school education (91\%) (Table 1). Over half of respondents had previously been diagnosed with cancers of the breast or ovary. Nonrespondents $(n=176)$ were younger than participants $(P=0.002)$, less educated $(P<$ $0.001)$, and less likely to have had cancer $(P<0.001)$. There was no difference between participants and nonresponders with respect to ethnicity or reported Jewish ancestry (Table 1).

Approximately $20 \%(N=63)$ of all respondents were categorized as high risk for having a BRCA mutation based on the Myriad Prevalence Tables ( $\geq 10 \%$ risk) (Table 2). The remaining $80 \%$ of women were about equally divided between the moderate risk $(N=122)$ and low-risk groups $(N=130)$. High-risk women tended to be younger, more educated, and more likely to be of Jewish ancestry than low-risk women. Risk groups were similar with respect to race/ethnicity. The vast majority of high- and moderate-risk women were affected, 
Table 1

Characteristics of study participants $(N=316)$

\begin{tabular}{|c|c|c|c|}
\hline Characteristics & $\begin{array}{l}\text { Respondents, } \\
N=315(\%)\end{array}$ & $\begin{array}{l}\text { Nonrespondents, } \\
\quad N=176(\%)\end{array}$ & $P$ \\
\hline \multicolumn{4}{|l|}{ Age } \\
\hline $25-39$ & 16 & 44 & 0.002 \\
\hline $40-49$ & 45 & 90 & \\
\hline $50-54$ & 39 & 42 & \\
\hline \multicolumn{4}{|l|}{ Race/ethnicity } \\
\hline Non-Hispanic white & 94 & 92 & 0.56 \\
\hline Hispanic & 4 & & 6 \\
\hline Other & 2 & & 2 \\
\hline \multicolumn{4}{|l|}{ Jewish ancestry } \\
\hline Yes & 3 & 2 & 0.22 \\
\hline No & 97 & & 98 \\
\hline \multicolumn{4}{|l|}{ Education level } \\
\hline High school or less & 9 & 17 & $<0.001$ \\
\hline$>$ High school & 91 & 83 & \\
\hline \multicolumn{4}{|l|}{ Affected status } \\
\hline Cancer & 65 & 34 & $<0.001$ \\
\hline No cancer & 35 & 66 & \\
\hline \multicolumn{4}{|l|}{ Cancer type (numbers) } \\
\hline Breast cancer & 187 & 53 & \\
\hline Ovarian cancer & 13 & 1 & \\
\hline Other cancer & 5 & 5 & \\
\hline No cancer & 110 & 117 & \\
\hline
\end{tabular}

whereas the majority of low-risk women $(73 \%)$ were not affected by cancer. Twenty-seven women reported having undergone genetic testing before the start of the ad campaign, which included 18 of 63 high-risk women (33\%), 7 moderate risk (6\%), and 2 low risk (2\%). Although testing could not be verified, we assumed that this was true and that these women had received additional information about genetic testing that may bias their responses to the survey so they were excluded from further analyses.

Over $70 \%$ of women said that they had heard of BRACAnalysis (Table 3). However, women at high or moderate risk were more likely to have heard of BRCAnalysis than were women at low risk ( 71 and $83 \%$, vs. $60 \% ; P=0.01$ ). High-risk women also considered themselves more knowledgeable about genetic testing than did low-risk women. Among those who had ever heard of BRACAnalysis, over $40 \%$ of all women said that they were interested in having the test. This proportion did not vary substantially by risk group.

Women at high and moderate genetic risk were more likely to recall having seen the advertisements for BRACAnalysis than were women at low risk $(60 \%, 57 \%$, vs. $39 \%, P<0.001)$ (Table 3). Among women who could recall having seen the ads
Table 2

Characteristics of survey respondents according to risk of BRCA mutation

\begin{tabular}{|c|c|c|c|c|}
\hline Characteristic & $\begin{array}{c}\text { Total } \\
(N)\end{array}$ & $\begin{array}{c}\text { High }=63 \\
(\%)\end{array}$ & $\begin{array}{c}\text { Moderate } \\
(\%)\end{array}$ & $\begin{array}{c}\text { Low }=130 \\
(\%)\end{array}$ \\
\hline Total & 315 & 20 & 39 & 41 \\
\hline \multicolumn{5}{|l|}{ Age group } \\
\hline $25-39$ & 51 & 16 & 14 & 18 \\
\hline $40-49$ & 122 & 62 & 45 & 38 \\
\hline $50-54$ & 142 & 22 & 41 & 44 \\
\hline \multicolumn{5}{|l|}{ Race/ethnicity } \\
\hline Non-Hispanic white & 296 & 94 & 91 & 97 \\
\hline Hispanic & 13 & 2 & 7 & 3 \\
\hline Other & 6 & 4 & 2 & 0 \\
\hline Jewish ancestry & 9 & 10 & 1 & 2 \\
\hline \multicolumn{5}{|l|}{ Education level } \\
\hline Less than high school & 24 & 3 & 4 & 13 \\
\hline High school or greater & 291 & 97 & 96 & 87 \\
\hline \multicolumn{5}{|l|}{ Cancer status } \\
\hline Breast cancer & 187 & 70 & 93 & 23 \\
\hline Ovarian cancer & 13 & 21 & 0 & 0 \\
\hline Other cancer & 5 & 0 & 0 & 4 \\
\hline Unaffected & 110 & 10 & 7 & 73 \\
\hline $\begin{array}{l}\text { Had testing before } \\
\text { campaign }\end{array}$ & 27 & 29 & 6 & 2 \\
\hline
\end{tabular}

(adding an additional 20 women who could recall the ad but only after interviewer prompted them about the nature and content of the ads), about $40 \%$ said that they were more interested in having the BRACAnalysis test after seeing the ad; this percentage was slightly higher in the high-risk group (48\% vs. $39 \%$ moderate risk vs. $32 \%$ low risk, NS). When asked how concerned they were about their personal risk for developing breast or ovarian cancer after seeing the ad, about $10 \%$ of women in each group reported feeling more concerned about their own risk; however, the majority of women stated that their concern remained the same as it was before seeing the ad.

Responses to survey questions intended to elicit more specific knowledge about the appropriateness and efficacy of genetic testing were fairly similar across risk groups, although there were some differences (Table 4). The majority of women, about $70 \%$, stated that they would want to know if they had the breast cancer gene. However, a lower percentage of low-risk women were confident they could get the BRACAnalysis test if they wanted it $(P<0.05)$. A similar percentage of high- and low-risk women thought that "because of their family history, they were likely to benefit from testing" (51\% high risk vs. 60\% low risk). Low-risk women were more likely than high-risk women to agree with the statement that "genetic testing benefits most women who have it" ( $64 \%$ vs. $40 \%$ of high risk, $P<$ 
Table 3

General knowledge of and interest in BRACAnalysis after seeing the Myriad ad campaign according to risk of BRCA mutation ${ }^{a}$

\begin{tabular}{|c|c|c|c|c|c|}
\hline Survey question & $P$ & & High $=45(\%)$ & Moderate $=115(\%)$ & Low $=128(\%)$ \\
\hline Have you ever heard of BRACAnalysis? & $<0.01$ & "Yes" & 71 & 83 & 60 \\
\hline If Yes, are you interested in having BRACAnalysis? & 0.79 & "Yes" & 47 & 43 & 49 \\
\hline \multicolumn{6}{|l|}{ What is your overall knowledge about genetic testing? } \\
\hline Know little or nothing & 0.01 & & 33 & 43 & 60 \\
\hline Know some thing & & & 60 & 52 & 38 \\
\hline Know a lot & & & 7 & 4 & 2 \\
\hline Do you recall seeing an ad for BRACAnalysis? & 0.01 & "Yes" & 60 & 57 & 39 \\
\hline If Yes, "after seeing the ad ... " were you more interested in having BRACAnalysis? & 0.68 & "Yes" & 48 & 39 & 32 \\
\hline \multicolumn{6}{|l|}{ How did you feel about your own risk for breast or ovarian cancer? } \\
\hline More concerned & 0.36 & & 11 & 10 & 13 \\
\hline Equally concerned & & & 85 & 74 & 76 \\
\hline Less concerned & & & 0 & 14 & 10 \\
\hline Do not know & & & 4 & 3 & 0 \\
\hline
\end{tabular}

${ }^{a}$ Total excludes 27 women (18 high, 7 moderate, 2 low risk) that reported having genetic testing before the ad campaign.

Table 4

Attitudes and beliefs about genetic testing by risk status (percent responding "Strongly Agree" or "Agree") ${ }^{a}$

\begin{tabular}{|c|c|c|c|}
\hline Statement & High $=45(\%)$ & Moderate $=115(\%)$ & Low $=128(\%)$ \\
\hline I would want to know if I had the breast cancer gene & 62 & 70 & 73 \\
\hline I feel confident that I could get the BRACAnalysis test if I wanted to & 91 & 87 & $77^{b}$ \\
\hline Given my family history, chances are low that I have the breast cancer gene & 33 & 48 & 31 \\
\hline Given my family history, I am likely to benefit from genetic testing & 51 & 46 & 60 \\
\hline Genetic testing for breast/ovarian cancer risk benefits most women who take it & 40 & 61 & $64^{b}$ \\
\hline Genetic testing for breast/ovarian cancer is useful for a small percent of women & 29 & 28 & $16^{b}$ \\
\hline Results from my genetic testing would also help my family know their risk & 69 & 82 & 79 \\
\hline Results from testing could help me prevent getting breast or ovarian cancer & 33 & 35 & 31 \\
\hline Genetic testing would tell me if I am going to get breast or ovarian cancer & 31 & 40 & 34 \\
\hline Results from testing would help me plan better for the future & 56 & 57 & 70 \\
\hline The results from genetic testing are very accurate & 36 & 45 & 38 \\
\hline I am worried about getting breast cancer ${ }^{c}$ & 47 & 37 & $73^{b}$ \\
\hline I am worried about getting ovarian cancer ${ }^{d}$ & 44 & 37 & 29 \\
\hline There is little I can do to change my risk for breast or ovarian cancer & 29 & 30 & 27 \\
\hline I am worried that testing would affect my health insurance or employment & 42 & 32 & $23^{b}$ \\
\hline
\end{tabular}

${ }^{a}$ Total excludes 27 women (18 high, 7 moderate, 2 low risk) that reported having genetic testing before the ad campaign.

${ }^{b} P<0.05$.

${ }^{c}$ Asked of nonbreast cancer cases only.

${ }^{d}$ Asked of nonovarian cancer cases only.

0.05). Low-risk women were also less likely to agree that "testing is only useful for a small percent of women" (16\% vs. 29\% high risk). Nearly $80 \%$ of all women felt that results from testing would help their family members. About one third of all women felt that genetic testing could predict cancer and that results from testing could help them prevent getting breast or ovarian cancer. Although a majority of respondents felt that testing results would help them plan for the future, less than half of women thought that the results from testing were accurate. Among unaffected women, a higher percentage of low-risk women were worried about getting breast cancer when compared with higher risk women $(P<0.05)$. Highrisk women were more worried that testing would affect their insurance. 
Table 5

Thoughts about genetic testing using BRACAnalysis according to risk group and ad recall

\begin{tabular}{|c|c|c|c|c|c|c|c|}
\hline & \multirow{2}{*}{$\frac{\text { Risk level }}{\text { Recall ad? (Total) }}$} & \multicolumn{2}{|c|}{ High } & \multicolumn{2}{|c|}{ Moderate } & \multicolumn{2}{|c|}{ Low } \\
\hline & & Yes (41) & No (22) & Yes $(70)$ & No $(52)$ & Yes (52) & No $(78)$ \\
\hline Have been tested & 34 & 16 & 5 & 7 & 3 & 2 & 1 \\
\hline Within past year & 7 & 1 & 1 & 2 & 1 & 0 & 1 \\
\hline More than a year ago & 27 & 15 & 4 & 5 & 2 & 2 & 0 \\
\hline Decided to be tested & 37 & 5 & 1 & 10 & 7 & 11 & 3 \\
\hline Made appt to be tested & 5 & 2 & 0 & 1 & 2 & 0 & 0 \\
\hline Decided not to be tested & 41 & 4 & 4 & 18 & 5 & 8 & 2 \\
\hline Undecided about testing & 121 & 13 & 5 & 39 & 9 & 40 & 15 \\
\hline Have discussed testing & 110 & 17 & 5 & 42 & 11 & 25 & 10 \\
\hline
\end{tabular}

Table reflects number or women responding "Yes."

In addition to the 27 women who reported having been tested before the campaign, seven women among the 315 interviewed $(2.2 \%)$ said that they had been tested by BRACAnalysis within the year after the ad campaign (Table 5). Another 37 women indicated that they had decided to get tested in the future (6 high-risk, 17 moderate risk, and 14 lowrisk women); however, only five of these women had made appointments to do so. Forty-one women had decided not to be tested and 121 women remained undecided. About $35 \%$ of women said that they had discussed genetic testing with someone since the time of the campaign, and this was more common among women that could recall having seen the ads across all risk groups.

\section{DISCUSSION}

The Myriad campaign was the first such mass media effort to promote genetic testing to the general population. Our data indicate that the campaign reached a large audience, and that women at increased genetic risk, who would be candidates for genetic testing, were more likely to recall having seen the ads than were women at lower risk. Nearly one half of high-risk women, who could recall seeing or hearing the ads, stated they were more interested in having testing, suggesting that the campaign may have impacted this population. However, that at least one third of low-risk women surveyed, who would not be considered at risk for having a $B R C A 1$ or $B R C A 2$ mutation, also expressed increased interest in testing, suggests that the ads may have been too broad in scope.

The current study was intended to parallel the CDC study aimed at assessing reaction to the campaign among a random sample of women living in the two campaign and control cities. ${ }^{7}$ Compared with the CDC Denver study, our respondents reported greater knowledge about BRACAnalysis (73\% vs. $45 \%)$ and interest in testing (46\% vs. $38 \%$ ). This is likely explained by the difference in patient populations between studies. By design, the CDC study focused primarily on averagerisk women, whereas our study targeted women with known genetic risk. Thus, heightened awareness about genetic testing in our respondents might be expected. With respect to ad recall and self-described knowledge about genetic testing, responses from the CDC Denver study are similar to those among our low-risk women; about $36 \%$ recalled the ad and about $60 \%$ report little or no knowledge about genetic testing. A higher proportion of respondents in our study said that they had discussed genetic testing with someone during the months after the campaign ( $34 \%$ vs. $8 \%$ ), and this was true even among our low-risk group, $26 \%$ of whom said they had discussed testing.

In the current study, we found that women were misinformed about the appropriateness of testing for themselves and others suggesting a need for more education surrounding this issue among all women. Of particular concern was the number of low-risk women who believed their family history made them candidates for testing. Only one third of low-risk women agreed that "the chance of them having a mutation was low." Furthermore, a similar proportion of high- and low-risk women (51 and 60\%) felt that because of their family history, they would benefit from testing. Although this is an appropriate response for women in the high-risk group, genetic testing would not be recommended for any women in the low-risk group. Current indications for referral for genetic testing generally follow the NCCN guidelines ${ }^{12}$ that recommend referral for women with a strong personal and/or family history of breast and ovarian cancer that is consistent with a $10 \%$ or greater risk for a $B R C A 1$ or $B R C A 2$ mutation by the Myriad tables. ${ }^{11}$ All women in our low-risk group had mutation probabilities of $<5 \%$ and, thus, would not be candidates for testing. Despite the fact that we did not also see an increase in the number of women who had sought testing after the campaign, several low-risk women indicated they had decided to pursue testing in the future thus the potential exists for an increase in demand for genetic services in this group. This was confirmed by a study conducted at Kaiser Permanente Colorado by Mouchawar et al. ${ }^{13}$ that reported an increase in genetic counseling referrals during and after the time of the Myriad campaign, the majority of which were for low-risk women. An 
increase in low-risk referrals may put a burden on the health care system by increasing demand for genetic services for which there is a limited pool of resources. Furthermore, increased demand services may delay services for those women at high risk who likely would benefit most from genetic counseling and genetic testing.

We also found that women across all risk groups overstated the benefits of genetic testing. Over one half of all women surveyed felt that genetic testing benefits most women, and less than one third agreed that testing is only useful for a small percentage of women. In reality, genetic testing is beneficial for a relatively small proportion of women with significant personal or family history of breast and/or ovarian cancer or who have a known mutation in their family. The latter likely constitutes $<1 \%$ of the general population. ${ }^{8}$ Furthermore, testing is most informative if done in an affected person. Testing in unaffected persons or in persons with low mutation risk often results in findings that are uninterpretable and, therefore, not useful to patients. The benefit of confirming mutation status among carriers is that these women and their physicians can make medical decisions to reduce their risk. Mutation carriers have a lifetime risk as high as $50-85 \%$ for breast cancer and $10-50 \%$ for ovarian cancer. ${ }^{5}$ Medical options for reducing risk include increased surveillance, chemoprevention, and prophylactic surgery. These surgical procedures can effectively reduce risk of breast and ovarian cancer by over $90 \% .^{14,15}$ In the current study, knowledge about the benefits of testing was limited across all risk groups (and in particular among low risk) and did not vary appreciably according to whether respondents could recall having seen the media ads (data not shown). This suggests that the campaign may not have impacted current knowledge about testing and further emphasizes the need for educational intervention among women for whom testing could be life altering.

We did not find that as a result of seeing the media ads, women were more concerned about their own risk for developing breast or ovarian cancer as was previously speculated. ${ }^{16}$ Only $10 \%$ of women in each risk group expressed increased concern. This finding is consistent with results from a similar study by Mouchawar et al. ${ }^{17}$ among 394 women enrolled in Kaiser Permanente Colorado. This study showed that the majority of women surveyed on their emotions after the Myriad media ads (63\%) reported no increase in anxiety. The results also showed that increased anxiety was associated with higher perceived risk. Because our survey did not specifically ask about perceived risk, we cannot directly compare these results. However, insomuch as risk group correlates with perceived risk, we could not confirm this in our population.

The BRACAnalysis campaign was widely defined as women aged 25-54 with a family history of breast or ovarian cancer. The television ad, which was the media most commonly recalled by our respondents, featured several women talking about their relative(s) with breast or ovarian cancer and about how BRACAnalysis would help them know their risk so that they could "be ready against cancer." The ad provided little detail about the types of families that might benefit from test- ing. It is conceivable that a woman with one relative with breast cancer diagnosed over 50 (who would have been included in our low-risk group) could have viewed the ad and considered herself at risk for having a BRCA mutation. In fact, about $20 \%$ of women in the target audience would be expected to have such a family history yet would not warrant testing. ${ }^{18}$ Furthermore, it was not clear that any of the women in the ad were affected with cancer themselves, whom as stated previously, would be the preferred person in the family to undergo testing. It is recognized that detail needed to adequately describe risk factors for and benefits of testing may not be feasible in 30second advertisement. However, a more targeted campaign addressing these issues may serve not only to dismiss fear among low-risk women but also trigger greater response and uptake of genetic testing among high-risk women who are not currently seeking these services.

One potential benefit to the BRACAnalysis campaign is that it increased awareness about the importance of family history in breast and ovarian cancer. Each year, about 200,000 cases of breast cancer and almost 25,000 cases of ovarian cancer are diagnosed in the United States. ${ }^{6}$ We still cannot explain the vast majority of these cancers. Between 3 and $4 \%$ of breast cancers are thought to be hereditary. ${ }^{2-4}$ Of those families with familial clustering of breast cancer, $<25 \%$ are explained by known breast cancer susceptibility loci. ${ }^{19}$ Other known risk factors including diet, reproductive history, and environmental exposure explain a very small proportion of cancers. Highlighting the importance of family history in assessing risk for cancer susceptibility may be beneficial. Increasing knowledge about risk may lead to greater uptake of information and screening for high-risk women, which may ultimately help reduce the burden of these cancers.

This study has some limitations that deserve mention. Although our study was strengthened by our ability to categorize women by risk based on family history information provided upon enrollment into CGN, we recognize that our study population may not be representative of the population targeted by the campaign. Individuals enrolled in the CGN represent a "higher than average" risk group as they either had cancer themselves or had a first-degree relative with cancer. Furthermore, by virtue of their enrollment into the registry, they are also likely a more motivated group with perhaps greater personal interest in issues surrounding cancer and genetics. Our response rate was reasonable for this type of study. However, nonrespondents tended to be younger and unaffected. Insomuch as these factors affect differences in response between risk groups, there is potential for selection bias. This study was also limited in our ability to conduct follow-up on subjects to verify reported testing or track referrals for testing. The survey was completed within 10 months of the start of the campaign, so it is possible that additional women sought testing that may have been inspired by the campaign beyond the survey period.

The Myriad campaign poses an important question: how do we inform the public about new technologies that enable us to identify individuals predisposed to disease without unnecessarily alarming those at low risk? The multimedia campaign for 
BRACAnalysis seemed to reach a very wide audience. Genetic testing is a promising tool for identifying those at risk and ultimately preventing disease. However, genetic information is complex, and as this survey has shown, is not well understood by most people. At present, those who stand to benefit from genetic testing comprise a very small fraction of the population. In 2007, Myriad relaunched the ad campaign in the northeastern region of the United States, focusing on Boston, Hartford, Providence, and New York City. ${ }^{20}$ The target audience remains the same, perhaps to maintain consistency with the 2002 campaign. It will be interesting to see what impact the campaign will have on this population. From our evaluation, if a media campaign is a viable approach to increasing awareness, a more targeted campaign aimed at those most likely to benefit from testing might be preferred.

\section{ACKNOWLEDGMENTS}

The authors acknowledge the Cancer Genetics Network, funding for which was provided by the National Cancer Institute (Grant No. CA078174); the Colorado Department of Public Health and Environment for their collaboration; and the Centers for Disease Control for use of their survey instrument.

\section{References}

1. Myriad Genetics Laboratories, Inc., 2002. Myriad genetics launches direct to consumer advertising campaign for breast cancer test. Available at: http://www.myriad.com/news/ release/333030. Accessed April 30, 2008.

2. Newman B, Mu H, Butler CM, Millikan RC, Moorman PG, King MC. Frequency of breast cancer attributable to BRCA1 in a population-based series of American women. JAMA 1998;279:915-921.

3. Peto J, Collins N, Barfoot R, et al. Prevalence of BRCA1 and BRCA2 gene mutations in patients with early-onset breast cancer. J Natl Cancer Inst 1999;91:943-949.
4. Syrjakoski K, Vahteristo P, Eerola H, et al. Population-based study of BRCA1 and BRCA2 mutations in 1035 unselected Finnish breast cancer patients. J Natl Cancer Inst 2000;92:1529-1531.

5. Easton DF, Ford D, Bishop DT, the Breast Cancer Linkage Consortium. Breast and ovarian cancer incidence in BRCA1 mutation carriers. Am J Hum Genet 1995;56:265-271.

6. Cancer Facts and Figures, 2006. Atlanta, GA: American Cancer Society. Available at: http://www.cancer.org. Accessed January 31, 2008.

7. Centers for Disease Control and Prevention (CDC). Genetic testing for breast and ovarian cancer susceptibility: evaluating direct-to-consumer marketing-Atlanta, Denver, Raleigh-Durham, and Seattle, 2003. MMWR Morb Mortal Wkly Rep 2004;53:603-606.

8. Parmigiani G, Berry DA, Aguilar O. Determining carrier probabilities for breast cancer-susceptibility genes BRCA1 and BRCA2. Am J Hum Genet 1998;62:145-158.

9. Nelson N. Cancer Genetics Network gets under way with 5 years of funding. J Natl Cancer Inst 1997;89:10-11.

10. Senior K. New US cancer genetics network announced. Mol Med Today 1998;4:459-460.

11. Myriad, 2003. Mutation prevalence tables. Available at: http://www.myriadtests.com/provider/mutprevo.htm. Accessed May 30, 2003.

12. National Comprehensive Cancer Network (NCCN), 2008. Available at: http://www.nccn. org/. Accessed August 29, 2008.

13. Mouchawar J, Hensley-Alford S, Laurion S, et al. Impact of direct-to-consumer advertising for hereditary breast cancer testing on genetic services at a managed care organization: a naturally-occurring experiment. Genet Med 2005;7:191-197.

14. Hartman LC, Schaid D, Sellers T, et al. Bilateral prophylactic mastectomy in BRCA1/2 mutation carriers. Proc Am Assoc Cancer Res 2001;41:222-223.

15. Rebbeck TR, Lynch HT, Neuhausen SL, Narod SA, et al. Prophylactic oophorectomy in carriers of BRCA1 and BRCA2 mutations. N Engl J Med 2002;346:1616-1622.

16. Gollust SE, Chandros Hull S, Wilfond BS. Limitations of direct-to-consumer advertising for clinical genetic testing. JAMA 2003;289:45-46.

17. Mouchawar J, Laurion S, Ritzwoller DP, Ellis J, et al. Assessing controversial direct-to-consumer advertising for hereditary breast cancer testing: reactions from women and their physicians in a managed care organization. Am J Manag Care 205;11:601-608.

18. Claus EB, Schildkraut JM, Thompson WD, Risch NJ. The genetic attributable risk of breast and ovarian cancer. Cancer 1996;77:2318-2324.

19. Easton DR, Pooley KA, Dunning AM, Pharoah PD, et al. Genome-wide association study identifies novel breast cancer susceptibility loci. Nature 2007;447: 1087-1095.

20. Myriad Genetics Laboratories, Inc., 2007. Myriad genetics launches awareness advertising campaign to educate women about hereditary risks of breast and ovarian cancers. Available at: http://www.myriad.com/news/release/1049527. Accessed May 30, 2008. 